\title{
FOXO3a-mediated suppression of the self-renewal capacity of sphere-forming cells derived from the ovarian cancer SKOV3 cell line by 7-difluoromethoxyl-5,4'-di-n-octyl genistein
}

\author{
YINGXIA NING ${ }^{1 *}$, CHAOYUAN LUO $^{2 *}$, KAIQUN REN $^{3}$, MEIFANG QUAN $^{3}$ and JIANGUO CAO ${ }^{3}$ \\ ${ }^{1}$ Department of Gynaecology and Obstetrics, The First Affiliated Hospital of Guangzhou Medical University, \\ Guangzhou 510120; ${ }^{2}$ Department of Oncological Surgery, The Third Affiliated Hospital of Guangzhou Medical University, \\ Guangzhou 510150; ${ }^{3}$ Laboratory of Medicine Engineering, Medical College, \\ Hunan Normal University, Changsha, Hunan 410013, P.R. China
}

Received July 31, 2013; Accepted February 13, 2014

DOI: $10.3892 / \mathrm{mmr} .2014 .2012$

\begin{abstract}
Carcinogenesis is predominantly dependent on the cancer stem cells (CSCs) residing or populating within the cancer. We previously demonstrated that the novel synthetic genistein analogue, 7-difluoromethoxyl-5,4'-di-n-octylgenistein (DFOG), induced apoptotic cell death of ovarian and gastric cancer cells. The present study demonstrated that sphere-forming cells (SFCs) derived from the ovarian cancer cell-line SKOV3 possessed ovarian cancer stem-like cell (OCSLC) properties, including self-renewal and high tumorigenicity. DFOG may be effective in inhibiting the self-renewal capacity of SFCs derived from the SKOV3 cell line. DFOG decreased the level of phosphorylated FOXO3a protein in SKOV3 cell-derived SFCs. The inhibition of FOXO3a expression by siRNA significantly attenuated the ability of DFOG to inhibit the self-renewal capacity of SKOV3-derived SFCs. Our results suggested that DFOG has been demonstrated to significantly inhibit the self-renewal capacity of ovarian cancer stem cells (OCSCs) through a mechanism partly dependent on the activation of FOXO3a.
\end{abstract}

\section{Introduction}

Ovarian cancer is the fourth leading cause of cancer-related mortality in females in the world and the leading cause of mortality from a gynecological cancer. The standard first-line treatment for ovarian cancer has not markedly altered since 1996 and includes the intravenous administration of a platinum agent

Correspondence to: Dr Jianguo Cao, Laboratory of Medicine Engineering, Medical College, Hunan Normal University, 371 Tongzipo Road, Yuelu, Changsha, Hunan 410013, P.R. China E-mail: caojianguo2005@126.com

*Contributed equally

Key words: ovarian cancer, cancer stem cells, self-renewal, 7-difluoromethoxyl-5,4'-di-n-octyl genistein, FOXO3a (carboplatin or cisplatin) and paclitaxel (Taxol) (1). Initially, the majority of patients respond, however, the disease usually recurs within 5 years. Thus, fewer than one in 10 patients survive beyond 5 years following standard salvage chemotherapy (2). Consequently, there is an urgent need to identify new and improved therapeutic approaches that are able to target this malignancy and improve long-term patient survival.

Cancer progression and development is predominantly dependent on the cancer stem cells (CSCs) residing or populating within the cancer. The self-renewing, near infinite proliferative capacity and potential for differentiation of CSCs is of vital importance in the occurrence, development and metastasis of cancer. It is believed that targeting CSCs may offer important and perhaps revolutionary advances in the targeting of cancer. Several dietary compounds, including curcumin $(3,4)$, quercetin (5,6), genistein (7-9), sulforaphane (10-14) and epigallocatechin-gallate $(15,16)$, may have potential therapeutic utility against CSC self-renewal. Previously, studies by our laboratory have demonstrated that the novel synthetic genistein analogue, 7-difluoromethoxyl-5,4'-di-n-octyl genistein (DFOG), induced cellular apoptotic death of ovarian and gastric cancer cells $(17,18)$. However, whether DFOG inhibits the selfrenewing capacity of ovarian cancer stem cells (OCSCs) has not been previously demonstrated.

The current study demonstrated that sphere-forming cells (SFCs) derived from the ovarian cancer cell-line SKOV3 possessed ovarian cancer stem-like cell (OCSLC) properties, including self-renewal and high tumorigenicity. For the first time, to the best of our knowledge, DFOG has been demonstrated to significantly inhibit the self-renewal capacity of OCSCs through a mechanism partly dependent on the activation of FOXO3a.

\section{Materials and methods}

Cell culture and reagents. The human ovarian cancer cell-lines, SKOV3, A2780 and OVCAR-3 were obtained from the cell bank of the Chinese Academy of Sciences (Shanghai, China). The cells were maintained as a monolayer in high glucose DMEM supplemented with $10 \%$ vol/vol fetal bovine 
serum (FBS), $100 \mathrm{IU} / \mathrm{ml}$ of penicillin $\mathrm{G}$ and $100 \mu \mathrm{g} / \mathrm{ml}$ of streptomycin at $37^{\circ} \mathrm{C}$ in a fully-humidified $5 \% \mathrm{CO}_{2}$ incubator. For sphere-forming culture, cells were collected and washed to remove serum, then suspended in serum-free DMEM/F12 supplemented with $100 \mathrm{IU} / \mathrm{ml}$ of penicillin, $100 \mu \mathrm{g} / \mathrm{ml}$ of streptomycin, $20 \mathrm{ng} / \mathrm{ml}$ of human recombinant epidermal growth factor, $10 \mathrm{ng} / \mathrm{ml}$ of human recombinant basic fibroblast growth factor, 2\% wt/vol B27 supplement without vitamin $\mathrm{A}$ and $1 \%$ wt/vol N2 supplement (Invitrogen Life Technologies, Carlsbad, CA, USA). The cells were subsequently cultured in ultra low attachment 6-well plates (Corning Inc., Corning, NY, USA) at a density of 1,000 cells/well. The culture medium was replaced or supplemented with additional growth factors twice a week. To propagate spheres in vitro, the cells were collected by gentle centrifugation, dissociated into single-cell suspensions and cultured to allow the regeneration of spheres. Third-generation spheres were used for all subsequent experiments.

In order to investigate the percentage of single cells capable of regenerating new spheres, cells were plated at a density of 1,000 cells $/ \mathrm{ml}$ in a 6 -well plate to obtain new spheres. The total number of tumor spheres was counted following 8 days of culture. The efficiency of sphere formation was calculated by dividing the total number of spheres formed by the total number of viable cells seeded and multiplying that result by a factor of 100 .

In vivo tumorigenicity experiments. All mice were cared for in accordance with the institutional guidelines of Hunan Normal University (Changsha, Hunan, China). The study was approved by the Ethics Committee of Hunan Normal University. Parental SKOV3 cells and the same cells at the third passage of SFC formation were used in tumorigenicity experiments. Trypan blue staining was used to assess cell viability and various numbers of single viable cells were subcutaneously injected into 4 week-old male BALB/c-nu mice (Shanghai Laboratory Animal Center, Chinese Academy of Sciences, Shanghai, China) in serum-free DMEM/Matrigel (at a 1:1 ratio) using a $100 \mu 1$ microsyringe. The mice were sacrificed 8 weeks following injection and tumors were harvested for further study.

MTT assay. The SFCs that were derived from the SKOV3 cell-line and parental cells were seeded at a density of 5,000 cells per well in 96-well plates that had been previously coated with Matrigel. The cells were treated with increasing concentrations of DFOG as indicated. Following $48 \mathrm{~h}$, MTT reagent (Sigma-Aldrich, St. Louis, MO, USA) was then added to each well according to the manufacturer's instructions. Absorbance values of each well were measured at $570 \mathrm{~nm}$.

Western immunoblot analysis. Western blot analysis was performed as previously described (18). The following primary antibodies were used: anti-FOXO3a, anti-p-FOXO3a, anti-Bmi1, anti-Nagon, anti-Sox2, anti-CD133, anti-CD44, anti-ALDH1 and anti- $\beta$-actin. The cells were lysed in a lysis buffer for $20 \mathrm{~min}$ at $4^{\circ} \mathrm{C}$. The protein concentration was determined using the Bio-Rad assay system (Bio-Rad, Hercules, CA, USA). Total proteins were fractionated by SDS-PAGE and transferred onto polyvinylidene fluoride membranes (Millipore, Billerica, MA, USA). The protein signals were detected by an ECL advance western blot system (Amersham Pharmacia Biotech Inc., Piscataway, NJ, USA).

Plasmids and transfections. A non-specific control siRNA (5'-UUCUCCGAACGUGUCACGUdTdT-3') was obtained from Qiagen (Hilden, Germany). FOXO3a siRNA (5'-ACUCCGGGUCCAGCUCCAC-3') was synthesized by Shanghai GenePharma Co., Ltd. (Shanghai, China). The transfection of cells with siRNA was performed using Lipofectamine 2000 (Invitrogen Life Technologies) according to the manufacturer's instructions. The cells were exposed to DMSO (control) or $10 \mu \mathrm{mol} / \mathrm{l}$ of DFOG for $24 \mathrm{~h}, 48 \mathrm{~h}$ after transfection. The cells were then collected and processed for western blot analysis and tumorsphere formation assay.

Statistical analysis. The database was set up with the SPSS 15.0 software package (SPSS, Inc., Chicago, IL, USA) for analysis. Data are presented as the mean \pm SD. The means of multiple groups were compared by one-way ANOVA, following confirmation of equal variance and pairwise comparisons among the means by the LSD method. Statistical comparisons were also made by the two-tailed t-test when appropriate. An $\alpha$ value of $\mathrm{P}<0.05$ was considered to indicate a statistically significant difference.

\section{Results}

Sphere formation and self-renewal in ovarian cancer cell lines. To determine if a population of self-renewing CSCs exists in ovarian cell-lines, SKOV3, OVCAR-3 and A2780 ovarian cancer cell-lines were grown in serum-free sphere-forming conditions. Following 8 days of culture, ball-like spheres ranging from 50 to 100 cells per sphere were observed. The cells from three cell lines formed spheres, however, at different sizes (Fig. 1A) and efficiencies (Fig. 1B), suggesting that each cell line contained a different number of cancer stem-like cells. SKOV3 cells exhibited a higher sphere-forming efficiency than OVCAR-3 and A2780 cells (Fig. 1B). Therefore, SKOV3 cells were selected for all further studies.

To assess the capability of SKOV3 cells to initiate self-renewal, they were subjected to several serial passages. SKOV3 spheres were dissociated into single cells and grown at a clonal density of 1,000 cells $/ \mathrm{ml}$. The dissociated first-generation spheres were able to generate second-generation spheres, which subsequently generated third-generation spheres (Fig. 1C). The sphere cultures were maintained for $>12$ passages, suggesting that SFCs that were derived from SKOV3 cells were fully capable of self-renewal. Thus, SKOV3 third-generation spheres were used in all subsequent experiments.

To determine the frequency of CSCs in SKOV3 third generation spheres, a limiting dilution assay was used to examine the ability of single cells from third-generation spheres to produce new spheres. Following 8 days of culture, 39.4\% of the single cells had generated new spheres. These spheres were able to be passaged $>12$ times. By contrast, a lower frequency of single cells derived from the parental cells was capable of regenerating spheres compared with single cells derived from third 
A

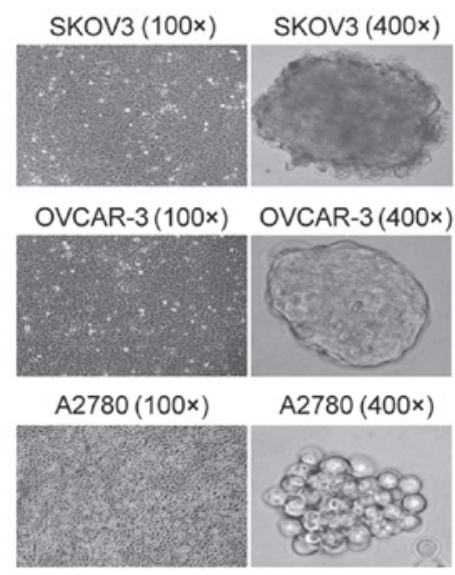

C

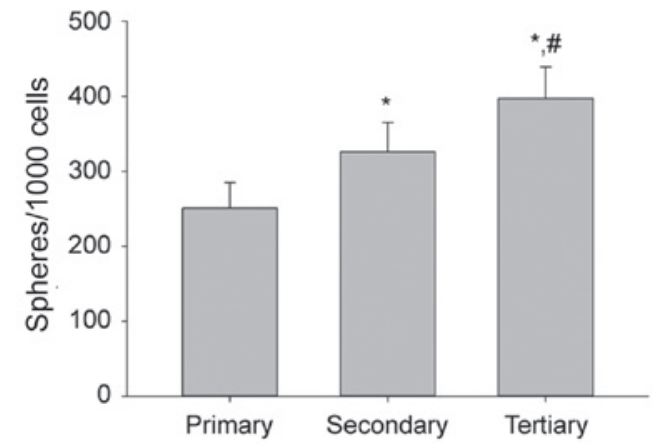

B

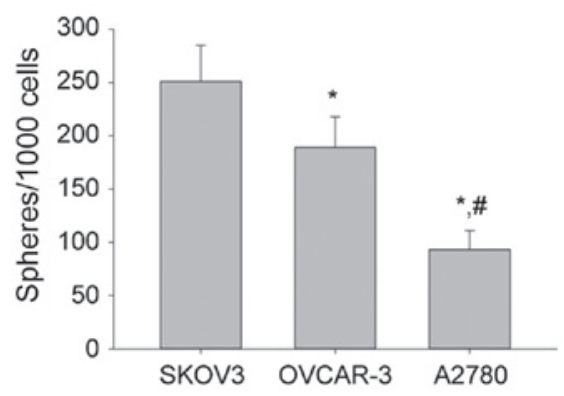

D

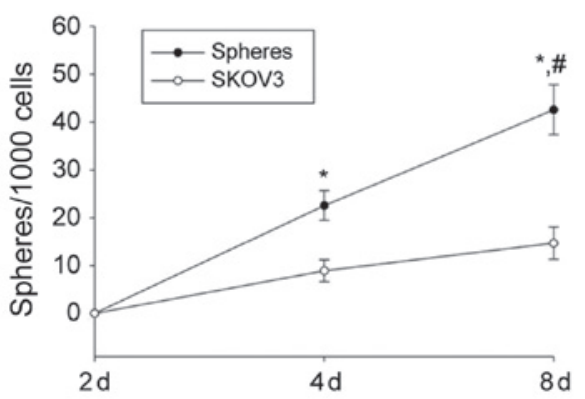

Figure 1. Sphere formation and self-renewal. (A) In a conditioned stem cell culture system, the human ovarian cancer cell lines SKOV3, OVCAR-3 and A2780 formed spheres and the volume of SFCs derived from SKOV3 cells was greater in size than that of either the OVCAR-3 or the A2780 cell-line. (B) Sphere-forming efficiency of SKOV3, OVCAR-3 and A2780 cells. The number of first-generation spheres formed on day 8 from 1,000 cells is shown. The data are presented as the mean \pm SD of three independent experiments. ${ }^{*} \mathrm{P}<0.05$, compared with SFCs derived from SKOV3 cells; ${ }^{*} \mathrm{P}<0.05$, compared with SFCs of OVCAR-3 cells. (C) Sphere-forming efficiency of SKOV3 during three serial passages. The number of primary, secondary (generated from dissociated primary spheres) and tertiary (generated from dissociated secondary spheres) spheres obtained from 1,000 cells is shown. The data are presented as the mean \pm SD of three independent experiments. "P<0.05, compared with primary spheres; ${ }^{*} \mathrm{P}<0.05$, compared with secondary spheres. (D) Number of spheres generated from tertiary spheres and from SKOV3 parental cells on the indicated days. Data are presented as the mean \pm SD of three independent experiments. ${ }^{*} \mathrm{P}<0.05$, compared with day 0 ; ${ }^{\#} \mathrm{P}<0.05$, compared with SKOV3 parental cells. SFCs, sphere-forming cells.

generation spheres (Fig. 1D). These results demonstrated that a considerable frequency of single cells that were derived from third generation spheres were self-renewing cells that were able to be expanded and maintained in culture as tumor spheres.

Enhanced expression of the self-renewal associated markers and tumorigenicity in SFCs derived from the SKOV3 cell line. To further characterize the expression profile of cell surface markers belonging to the SFCs, western blot analysis of several candidate self-renewal and stem cell associated markers was performed. BMI1, Nagon and SOX2 populations were enriched in tumor spheroids derived from SKOV3 cells (Fig. 2A). The present study also demonstrated enrichment of $\mathrm{CD} 33^{+}, \mathrm{CD}_{4} 4^{+}$and $\mathrm{ALDH}^{\text {high }}$ populations in SFCs derived from SKOV3 cells (Fig. 2B).

To test the tumorigenic potential of cells grown under sphere-forming conditions with enriched stem cells, SFCs of the SKOV3 cell line and parental cells at varying cell numbers were subcutaneously implanted in the flanks of nude mice. Tumors were able to be formed with only $10^{3}$ sphere cells, however, a minimum of $10^{6}$ parental cells was required to form xenograft tumors (Table I). The tumor nodules formed by SFCs of the SKOV3 cell line exhibited a similar histology to that observed by the parental cells (Fig. 2C). The results suggested that the tumorigenic efficacies of SFCs were enhanced compared with the parental cells and non-adherent tumor spheres that were derived from ovarian cancer SKOV3 cells cultured in stem cell conditioned medium, which also possessed properties of OCSLCs.

DFOG significantly reduces the formation of primary and secondary tumor spheroids in SFCs of the SKOV3 cell line. It has previously been reported that CSCs are capable of extensive proliferation. Genistein inhibited the proliferative activity of pancreatic CSCs $(8,9)$. The present study sought to examine whether DFOG inhibits SKOV3 cell-derived SFCs. Parental cells or third-generation spheres derived from SKOV3 cells were treated with various doses of DFOG (0.0$10.0 \mu \mathrm{M}$ ) for $48 \mathrm{~h}$. Cell viability was measured by an MTT assay. DFOG preferentially inhibited cell viability of SKOV3 cell-derived SFCs in a dose-dependent manner (Fig. 3A) and the $\mathrm{IC}_{50}$ values of DFOG against parental cells and SKOV3-derived SFCs were 10.90 and $0.47 \mu \mathrm{mol} / 1$, respectively. This observation suggested that DFOG was capable of preferentially suppressing the proliferation activity of OCSLCs. 
A

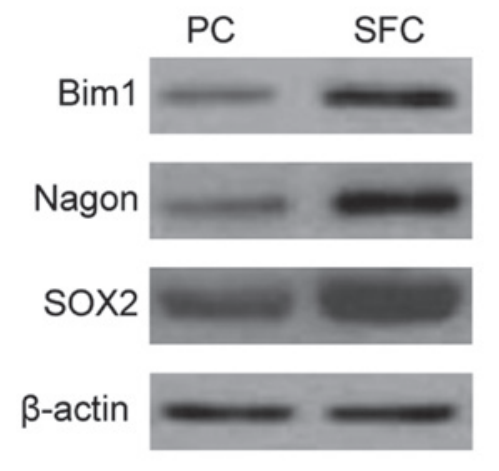

B

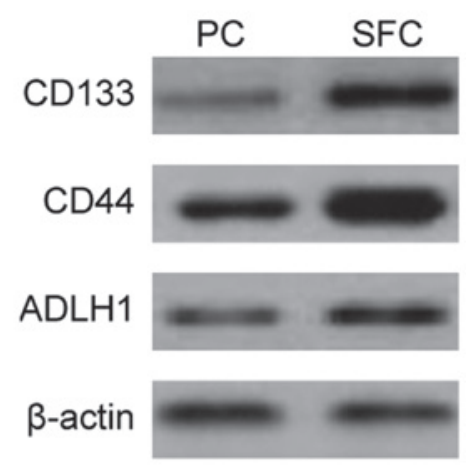

C

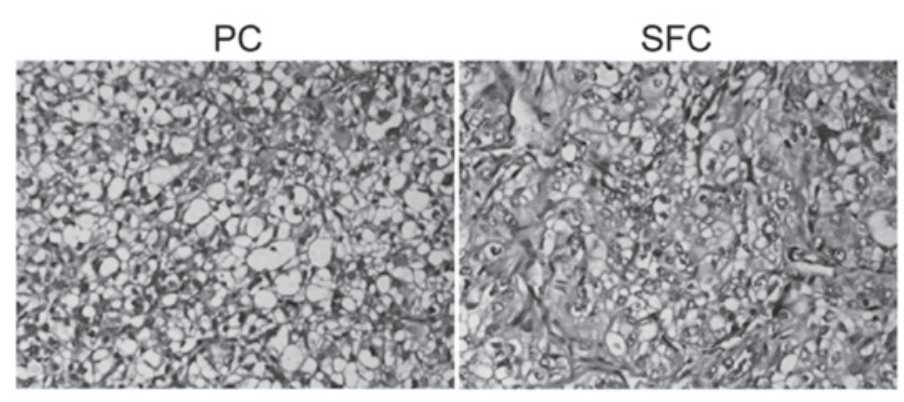

Figure 2. SKOV3 cell-line-derived SFCs possess properties of OCSLCs. (A) Western immunoblot analysis demonstrated that BMI1, Nagon and SOX2 were highly expressed in SFCs derived from SKOV3 cells compared with the PC. (B) Western immunoblot analysis demonstrated that CD133, CD44 and ADLH1 were highly expressed in SFCs derived from SKOV3 cells compared with the PC. (C) H\&E staining revealed that the histological features of SFC-derived xenografted tumors were similar to those identified for parental SKOV3 cells (magnification, x100). SFCs, sphere-forming cells; OCSLCs, ovarian cancer stem-like cells; PC, parental cells.

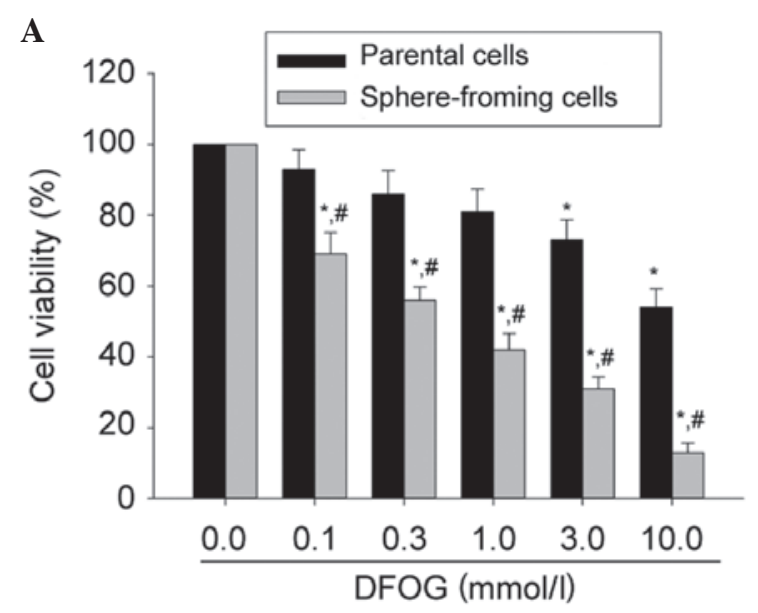

B

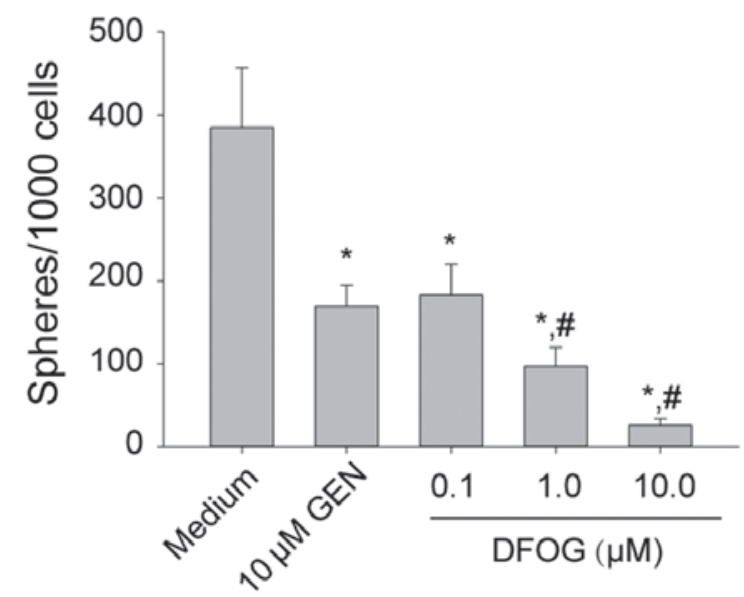

Figure 3. DFOG preferentially inhibits the proliferation and self-renewal of OCSLCs derived from SKOV3 cells. (A) DFOG preferentially inhibited the proliferation of SFCs derived from SKOV3 cells. (B) SFCs were incubated with varying concentrations of casticin (0.1-10.0 $\mu$ mol/1) or DMSO for 6 days. DFOG inhibited the self-renewal capacity of SFCs derived from SKOV3 cells. Data represent the mean \pm SD $(n=3)$. ${ }^{*} \mathrm{P}<0.05$ vs. treatment with the corresponding concentration of $0.1 \%$ DMSO in the treatment group; ${ }^{~} \mathrm{P}<0.05$ vs. parental cells treated with the corresponding concentration of casticin or $10.0 \mu \mathrm{mol} / 1$ of genistein. DFOG, 7-difluoromethoxyl-5,4'-di-n-octyl genistein; OCSLCs, ovarian cancer stem-like cells; SFCs, sphere-forming cells.

In order to determine whether DFOG suppressed the formation of tumor spheres in vitro, SKOV3 cell-derived SFCs were exposed to varying concentrations of DFOG or genistein $(10.0 \mu \mathrm{M} ;$ Fig. 3B). It was demonstrated that DFOG and genistein inhibited the formation of spheres. It is worth noting that the concentrations of DFOG that were capable of suppressing tumorsphere formation $\left(\mathrm{IC}_{50}\right.$ around $0.5 \mu \mathrm{M}$ for the SKOV3 spheres) were $\sim 10$-fold lower than those exhib- iting anti-proliferative effects by the MTT assay (with an $\mathrm{IC}_{50}$ around $10 \mu \mathrm{M}$ for parental SKOV3 cells). These data suggest that DFOG may be effective in inhibiting the self-renewal capacity of OCSLCs.

DFOG decreases the phosphorylation level of FOXO3a and stem cell surface markers of SKOV3 cell-derived SFCs. FOXO3a has been reported to be pivotal in the control of the 
Table I. Tumorigenicity experiments using SFCs derived from the SKOV3 cell line and parental cells in BALB/c-nu mice.

\begin{tabular}{|c|c|c|c|}
\hline Cell type & Cell number & Incidence & Latency (days) \\
\hline \multirow[t]{5}{*}{ Parental cells } & $5 \times 10^{4}$ & $0 / 4$ & - \\
\hline & $1 \times 10^{5}$ & $0 / 4$ & - \\
\hline & $2 \times 10^{5}$ & $3 / 4$ & 35 \\
\hline & $5 \times 10^{5}$ & $4 / 4$ & 29 \\
\hline & $1 \times 10^{6}$ & $4 / 4$ & 12 \\
\hline \multirow[t]{5}{*}{$\mathrm{CD}_{133^{+}}$cells } & $5 \times 10^{2}$ & $0 / 4$ & - \\
\hline & $1 \times 10^{3}$ & $4 / 4$ & 25 \\
\hline & $5 \times 10^{3}$ & $4 / 4$ & 13 \\
\hline & $1 \times 10^{4}$ & $4 / 4$ & 9 \\
\hline & $5 \times 10^{4}$ & $4 / 4$ & 6 \\
\hline
\end{tabular}

SFCs, sphere-forming cells.

A

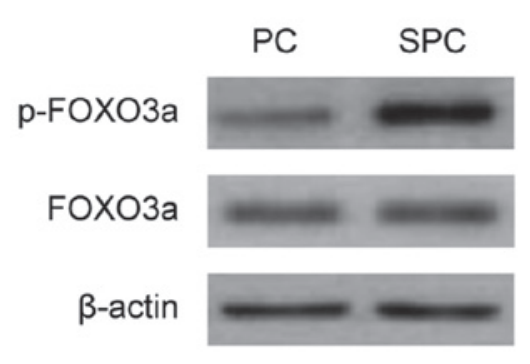

C

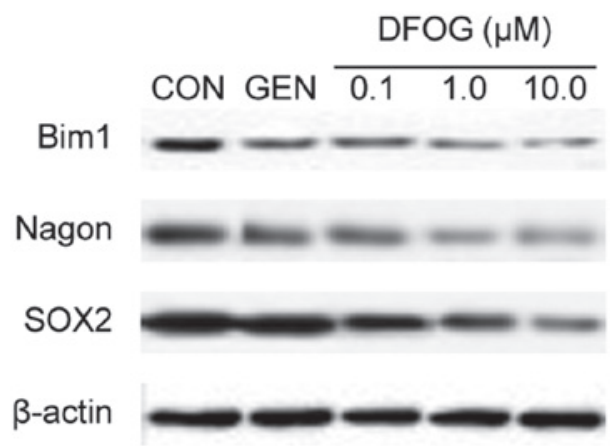

B

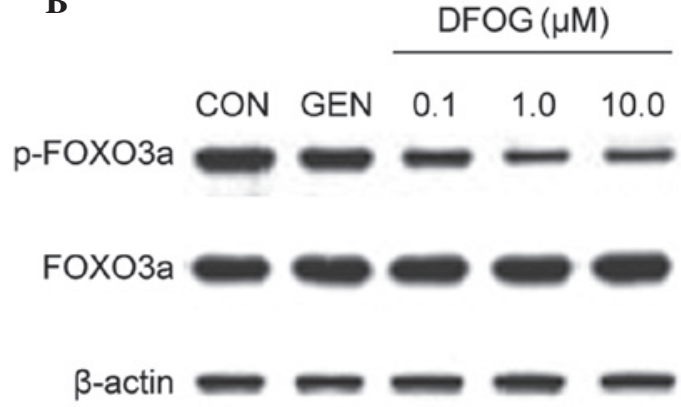

D

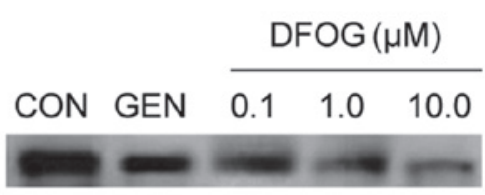

CD44

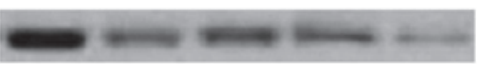

ALDH1

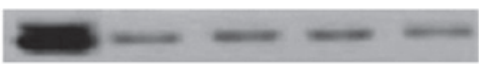

$\beta$-actin

Figure 4. DFOG reduces FOXO3a phosphorylation and CSC marker expression of OCSLCs derived from SKOV3 cells. (A) The phosphorylated form of the FOXO3a protein was highly expressed in SFCs derived from SKOV3 cells compared with corresponding PCs. (B) Treatment with DFOG downregulated the expression of phosphorylated FOXO3a in SFCs derived from SKOV3 cells. (C) Treatment with DFOG downregulated the expression of the self-renewal associated proteins, including BMI, Nagon and SOX2 in SFCs derived from SKOV3 cells. (D) Treatment with DFOG downregulated the expression of CSC markers, including CD133, CD44 and ALDH1 in SFCs derived from SKOV3 cells. DFOG, 7-difluoromethoxyl-5,4'-di-n-octyl genistein; CSC, cancer stem cell; OCSLCs, ovarian cancer stem-like cells; SFCs, sphere-forming cells; PCs, parental cells.

tumorigenicity of glioblastoma CSLCs (19). Thus, the present study sought to investigate if DFOG inhibited the self-renewal capacity of SFCs derived from the SKOV3 cell-line and whether DFOG was involved in the modulation of FOXO3a activity. SKOV3-derived third generation spheres elevated the protein expression levels of phosphorylated FOXO3a, indicating the presence of FOXO3a inactivation (Fig. 4A). In addition, DFOG decreased the level of phosphorylated FOXO3a protein in SKOV3 cell-derived SFCs (Fig. 4B). These data suggest that DFOG decreased the activity of FOXO3a and that it may participate in inhibiting the self-renewal capacity of OCSLCs.

The study by Shiota et al demonstrated that FOXO3a regulates the motility of urothelial cancer cells through negative regulation of Twistl (20). Previous demonstration of 
A

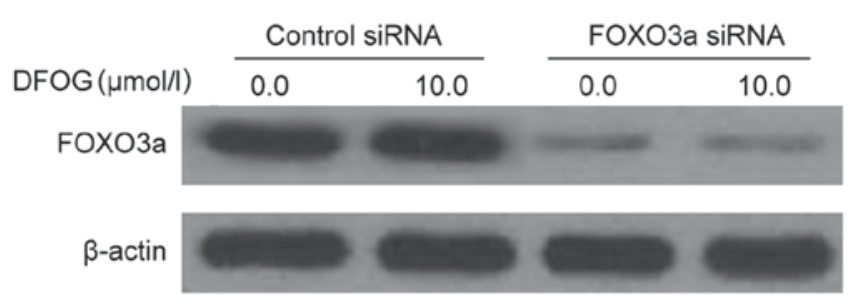

B

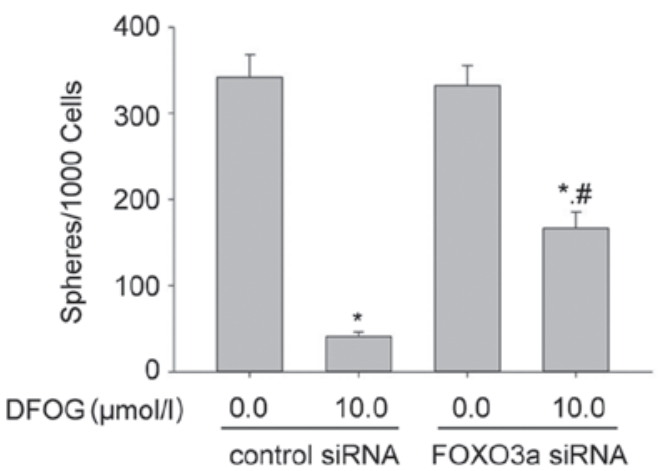

Figure 5. Silencing of FOXO3a attenuates the inhibitory effect of casticin on the self-renewal capacity of OCSLCs derived from SKOV3 cells. (A) Western blot analysis demonstrated silencing of FOXO3a following transfection of SFCs derived from SKOV3 cells with FOXO3a siRNA. Gene silencing of FOXO3a reduced FOXO3a expression. (B) Sphere forming ability of SFCs derived from SKOV3 cells that were treated with DFOG, were partly rescued following gene silencing of FOXO3a. Data represent the mean \pm SD $(n=3)$. ${ }^{*} \mathrm{P}<0.05$ vs. $0.1 \%$ DMSO treated cells. ${ }^{*} \mathrm{P}<0.05$ vs. the cells treated with the same concentration of DFOG using control siRNA transfection. DFOG 7-difluoromethoxyl-5,4'-di-n-octyl genistein; OSCLCs, ovarian cancer stem-like cells; SFCs, sphere-forming cells.

Twist1 directly regulating the expression of BMI1 provides a mechanistic explanation of the association between EMT and cancer stemness (21). The present study aimed to investigate whether DFOG affected the expression of BMI1, Nagon and SOX2 proteins in OCSLCs. Fig. 4C shows that DFOG reduced the protein expression levels of BMI1, Nagon and SOX2 in SKOV3-derived SFCs.

Since inactivation of FOXO3a led to an increase in SFC capacity and expression of the CSC surface marker CD44 in prostate cancer stem-like cell populations (22), the present study next analyzed whether DFOG inhibited the protein expression levels of CD133, CD44 and ALDH1. DFOG inhibited the protein expression levels of CD133, CD44 and ALDH1 in SKOV3-derived SFCs (Fig. 4D). These results illustrated that DFOG downregulated the expression of CD133, CD44 and ALDH1 in OCSLCs.

Knockout of $\mathrm{FOXO} 3$ a attenuates the inhibitory effects of DFOG on the self-renewal capacity of SKOV3-derived $S F C s$. The present study examined whether activation of FOXO3a affected DFOG-inhibited self-renewal capacity of SKOV3-derived SFCs. DFOG and genistein inhibited the self-renewal capacity in SFCs/control siRNA cells that were derived from SKOV3 cells. The inhibition of FOXO3a expression by siRNA significantly attenuated the ability of DFOG to inhibit the self-renewal capacity of SKOV3-derived SFCs
(Fig. 5A and B). These data suggest that the silencing of FOXO3a contributed to the self-renewal capacity of OCSLCs.

\section{Discussion}

The anti-cancer efficacy of DFOG, which is a novel synthetic analogue of genistein, has been evaluated in several types of cancer, including ovarian cancer cell lines. For instance, DFOG inhibited the growth and induced apoptosis of gastric cancer and ovarian cancer cells $(17,18)$. Increasing evidence supports the cancer stem cell theory, which hypothesizes that various types of cancer are driven and maintained by a small proportion of CSCs (23). The concept of CSCs has profound clinical implications for cancer therapeutics, management and prevention $(23,24)$. Previous studies indicated that CSCs have the capacity to drive tumor resistance and relapse/recurrence $(25,26)$. Since ovarian cancer lacks efficacy or sensitivity to the effects of current chemotherapies, metastatic disease requires novel approaches to specifically target CSC populations $(23,27,28)$. Numerous studies found that several dietary compounds are promising chemopreventive agents against CSCs, including genistein (7-9). Therefore, based on the chemopreventive activity of genistein and DFOG, and the implications of the CSC theory, the present study determined whether DFOG acted against OCSCs.

Numerous techniques have been developed to isolate and characterize OCSCs in vitro. Tumorsphere cultures were first used to isolate and expand ovarian cancer stem/progenitor cells $(29,30)$, and were based on the ability of stem/progenitor cells to grow in serum-free suspension, while differentiated cells failed to survive under the same conditions $(29,30)$. By employing this technique, the present study demonstrated that SKOV3 cell-derived SFCs possessed the properties of OCSLCs, including the self-renewal capacity and higher tumorigenicity.

The well-known effects of genistein were also demonstrated to regulate self-renewal associated transcription factors in previous studies, including Nagon (31), Bmi1 (30) and Sox 2 (32). The present study for the first time, to the best of our knowledge, provided evidence that DFOG preferentially inhibited the cellular viability and self-renewal capacity of SFCs derived from ovarian cancer SKOV3 cells. This observed effect was also accompanied by downregulation of the self-renewal associated transcription factors, Bmi1, Nagon and Sox 2 protein expression. The preference of DFOG in inhibiting CSCs may be significant for chemopreventive effects.

The study by Sunayama et al demonstrated that FOXO3a may function as a key integrator of these cellular signals that regulate glioblastoma CSLCs and which may also be considered a potential therapeutic target in the treatment of glioblastoma (19). The present study demonstrated that DFOG activated FOXO3a via inhibiting phosphorylation of the FOXO3a protein in SFCs derived from SKOV3 cells. Silencing of the FOXO3a gene by transfection with FOXO3a siRNA attenuated the ability of DFOG to inhibit the self-renewal capacity of SKOV3 cell-derived SFCs. The significance of the results indicates that not only is the DFOG-inhibitory effect of the self-renewal capacity associated with the activation of FOXO3a, but also the inactivation of FOXO3a contributes to the self-renewal capacity of SKOV3 cell-derived SFCs. 
In conclusion, the present study demonstrated that DFOG was able to target OCSLCs as determined by the tumorsphere formation assay. Furthermore, the present study identified the activation of FOXO3a by DFOG as one of the possible mechanisms for its efficacy. These studies support the use of DFOG for ovarian cancer chemoprevention. These findings provide a persuasive and supporting rationale for the preclinical and clinical evaluation of DFOG targeted therapy of ovarian cancer.

\section{Acknowledgements}

This study was supported by the National Natural Science Foundation of China (no. 81301894), Guangdong Province Science and Technique Department Item (no.2012B031800271) of China and Guangzhou Science and Information Bureau Item (20130000015) of China.

\section{References}

1. Jemal A, Center MM, Ward E and Thun MJ: Cancer occurrence. Methods Mol Biol 471: 3-29, 2009.

2. Schwartz PE: Current diagnosis and treatment modalities for ovarian cancer. Cancer Treat Res 107: 99-118, 2002.

3. Almanaa TN, Geusz ME and Jamasbi RJ: Effects of curcumin on stem-like cells in human esophageal squamous carcinoma cell lines. BMC Complement Altern Med 12: 195, 2012.

4. Quitschke WW: Curcuminoid binding to embryonal carcinoma cells: reductive metabolism, induction of apoptosis, senescence, and inhibition of cell proliferation. PLoS One 7: e39568, 2012.

5. Chen SF, Nieh S, Jao SW, et al: Quercetin suppresses drug-resistant spheres via the p38 MAPK-Hsp27 apoptotic pathway in oral cancer cells. PLoS One 7: e49275, 2012.

6. Lee $\mathrm{CH}$, Hong HM, Chang YY and Chang WW: Inhibition of heat shock protein (Hsp) 27 potentiates the suppressive effect of Hsp90 inhibitors in targeting breast cancer stem-like cells. Biochimie 94: 1382-1389, 2012.

7. Montales MT, Rahal OM, Kang J, et al: Repression of mammosphere formation of human breast cancer cells by soy isoflavone genistein and blueberry polyphenolic acids suggests diet-mediated targeting of cancer stem-like/progenitor cells Carcinogenesis 33: 652-660, 2012.

8. Bao B, Wang Z, Ali S, et al: Over-expression of FoxM1 leads to epithelial-mesenchymal transition and cancer stem cell phenotype in pancreatic cancer cells. J Cell Biochem 112: 2296-2306, 2011.

9. Bao B, Wang Z, Ali S, et al: Notch-1 induces epithelial-mesenchymal transition consistent with cancer stem cell phenotype in pancreatic cancer cells. Cancer Lett 307: 26-36, 2011.

10. Rodova M, Fu J, Watkins DN, Srivastava RK and Shankar S: Sonic hedgehog signaling inhibition provides opportunities for targeted therapy by sulforaphane in regulating pancreatic cancer stem cell self-renewal. PLoS One 7: e46083, 2012.

11. Kallifatidis G, Labsch S, Rausch V, et al: Sulforaphane increases drug-mediated cytotoxicity toward cancer stem-like cells of pancreas and prostate. Mol Ther 19: 188-195, 2011.

12. Rausch V, Liu L, Kallifatidis G, et al: Synergistic activity of sorafenib and sulforaphane abolishes pancreatic cancer stem cell characteristics. Cancer Res 70: 5004-5013, 2010.
13. Li Y, Zhang T, Korkaya H, et al: Sulforaphane, a dietary component of broccoli/broccoli sprouts, inhibits breast cancer stem cells. Clin Cancer Res 16: 2580-2590, 2010.

14. Chaudhuri D, Orsulic S and Ashok BT: Antiproliferative activity of sulforaphane in Akt-overexpressing ovarian cancer cells. Mol Cancer Ther 6: 334-345, 2007.

15. Chen D, Pamu S, Cui Q, Chan TH and Dou QP: Novel epigallocatechin gallate (EGCG) analogs activate AMP-activated protein kinase pathway and target cancer stem cells. Bioorg Med Chem 20: 3031-3037, 2012.

16. Nishimura N, Hartomo TB, Pham TV, et al: Epigallocatechin gallate inhibits sphere formation of neuroblastoma $\mathrm{BE}(2)-\mathrm{C}$ cells. Environ Health Prev Med 17: 246-251, 2012.

17. Xiang HL, Liu F, Quan MF, Cao JG and Lv Y: 7-difluoromethoxyl-5,4'-di-n-octylgenistein inhibits growth of gastric cancer cells through downregulating forkhead box M1. World J Gastroenterol 18: 4618-4626, 2012.

18. Ning Y, Li Q, Xiang H, Liu F and Cao J: Apoptosis induced by 7-difluoromethoxyl-5,4'-di-n-octyl genistein via the inactivation of FoxM1 in ovarian cancer cells. Oncol Rep 27: 1857-1864, 2012.

19. Sunayama J, Sato A, Matsuda K, et al: FoxO3a functions as a key integrator of cellular signals that control glioblastoma stem-like cell differentiation and tumorigenicity. Stem Cells 29: 1327-1337, 2011.

20. Shiota M, Song Y, Yokomizo A, et al: Foxo3a suppression of urothelial cancer invasiveness through Twist1, Y-box-binding protein 1, and E-cadherin regulation. Clin Cancer Res 16: 5654-5663, 2010

21. Wu KJ and Yang MH: Epithelial-mesenchymal transition and cancer stemness: the Twist1-Bmil connection. Biosci Rep 31: 449-455, 2011.

22. Dubrovska A, Kim S, Salamone RJ, et al: The role of PTEN/Akt/PI3K signaling in the maintenance and viability of prostate cancer stem-like cell populations. Proc Natl Acad Sci USA 106: 268-273, 2009.

23. Reya T, Morrison SJ, Clarke MF and Weissman IL: Stem cells, cancer, and cancer stem cells. Nature 414: 105-111, 2001.

24. Kakarala M and Wicha MS: Implications of the cancer stem-cell hypothesis for breast cancer prevention and therapy. J Clin Oncol 26: 2813-2820, 2008.

25. Sakariassen $P \emptyset$, Immervoll $\mathrm{H}$ and Chekenya $\mathrm{M}$ : Cancer stem cells as mediators of treatment resistance in brain tumors: status and controversies. Neoplasia 9: 882-892, 2007.

26. Tang C, Chua CL and Ang BT: Insights into the cancer stem cell model of glioma tumorigenesis. Ann Acad Med Singapore 36: 352-357, 2007.

27. Lippman ME: High-dose chemotherapy plus autologous bone marrow transplantation for metastatic breast cancer. N Engl J Med 342: 1119-1120, 2000.

28. Williams SD, Birch R, Einhorn LH, Irwin L, Greco FA and Loehrer PJ: Treatment of disseminated germ-cell tumors with cisplatin, bleomycin, and either vinblastine or etoposide. N Engl J Med 316: 1435-1440, 1987.

29. Liu T, Cheng W, Lai D, Huang Y and Guo L: Characterization of primary ovarian cancer cells in different culture systems. Oncol Rep 23: 1277-1284, 2010.

30. Ma L, Lai D, Liu T, Cheng W and Guo L: Cancer stem-like cells can be isolated with drug selection in human ovarian cancer cell line SKOV3. Acta Biochim Biophys Sin (Shanghai) 42: 593-602, 2010.

31. Li Y, Chen H, Hardy TM and Tollefsbol TO: Epigenetic regulation of multiple tumor-related genes leads to suppression of breast tumorigenesis by dietary genistein. PLoS One 8: e54369, 2013.

32. Regenbrecht CR, Jung M, Lehrach $\mathrm{H}$ and Adjaye J: The molecular basis of genistein-induced mitotic arrest and exit of self-renewal in embryonal carcinoma and primary cancer cell lines. BMC Med Genomics 1: 49, 2008. 